\title{
Two interesting cases of oddball AGN
}

\author{
Michael J. Ledlow ${ }^{1}$, Frazer N. Owen ${ }^{2}$ \\ and William C. Keel ${ }^{3}$ \\ ${ }^{1}$ Gemini Observatory, Southern Operations Center, AURA/Casilla 603, La Serena, Chile \\ email: mledlow@gemini.edu \\ ${ }^{2}$ National Radio Astronomy Observatory, Soccoro, NM 87801, USA \\ ${ }^{3}$ Department of Physics \& Astronomy, University of Alabama, Tuscaloosa, AL 35487, USA
}

\begin{abstract}
We present new observations from Gemini-South for two unusual AGNs: the recentlydiscovered, largest-known FR I radio galaxy $(>1.1 \mathrm{Mpc})$, and a FR I radio galaxy originating from a spiral host (the wrong kind of galaxy!). Both AGNs may represent missing classes of objects, not present in current surveys and samples.
\end{abstract}

\section{The Largest FRI Radio Galaxy}

As part of a large study of cluster and non-cluster radio galaxies, the radio galaxy B2 $1108+27$, was found to be much more extended than previously thought. With FIRST, B2 $1108+27$ appears as a naked-jet source with a full extent of only $44 \mathrm{kpc}$. Deeper imaging with the VLA at $20 \mathrm{~cm}$ in the D-array has confirmed that the source is considerably more extended; $\geqslant 1.1 \mathrm{Mpc}$ (see Figure 1), thus falling within the class of giant radio galaxies. B2 $1108+27$ is unique, however, in that the radio luminosity is an order of magnitude less than the lowest luminosity giant radio galaxies currently known, and is also of FR I morphology.

In figure 1, we show followup optical imaging with Gemini-South and $H \alpha$ imaging from Cerro Tololo. These data reveal that B2 1108+27 shares similar morphological properties to Centaurus-A, and hence is most likely the result of a major merger. Yet such a violent environment would seem to be the last place that one should find a giant low-luminosity radio galaxy with extremely straight and undisturbed jets. A recent Gemini+GMOS longslit spectrum has revealed a central dust-disk in scattered light from a powerful AGN. The implied mass is $4 \times 10^{10} M_{\odot}$ within $2 \mathrm{kpc}$ and $2 \times 10^{11} M_{\odot}$ within $6 \mathrm{kpc}$, pointing to a very large central BH mass (possibly a binary)?

\section{A Unique Spiral Radio Galaxy}

The discovery of the spiral-host radio galaxy 0313-192 was reported in Ledlow et al. (1998), Ledlow et al. (2001). Here, we present new images (see also: Keel et al. (2002)) from HST and Gemini, illustrating beyond doubt the spiral ( $\mathrm{Sa} / \mathrm{Sb}$ ) nature of the host and clearly showing a warped disk. From narrow-band HST imaging, we also find evidence of an inner, tilted disk, possibly indicating an interaction or minor merger. We have attempted an indirect measurement of the $\mathrm{BH}$ mass from the dispersion-width of the CaTriplet with Gemini, but the results are inconclusive. This object makes an interesting test-case for understanding the relationship between $\mathrm{BH}$ mass and nuclear activity. Based on bulge luminosity, we might expect a $\mathrm{BH}$ mass of $\geqslant 10^{8} M_{\odot}$, however the spiral nature of the host places it in a relatively unsampled region of the $M_{B H} / M_{\text {bulge }}$ plane. 

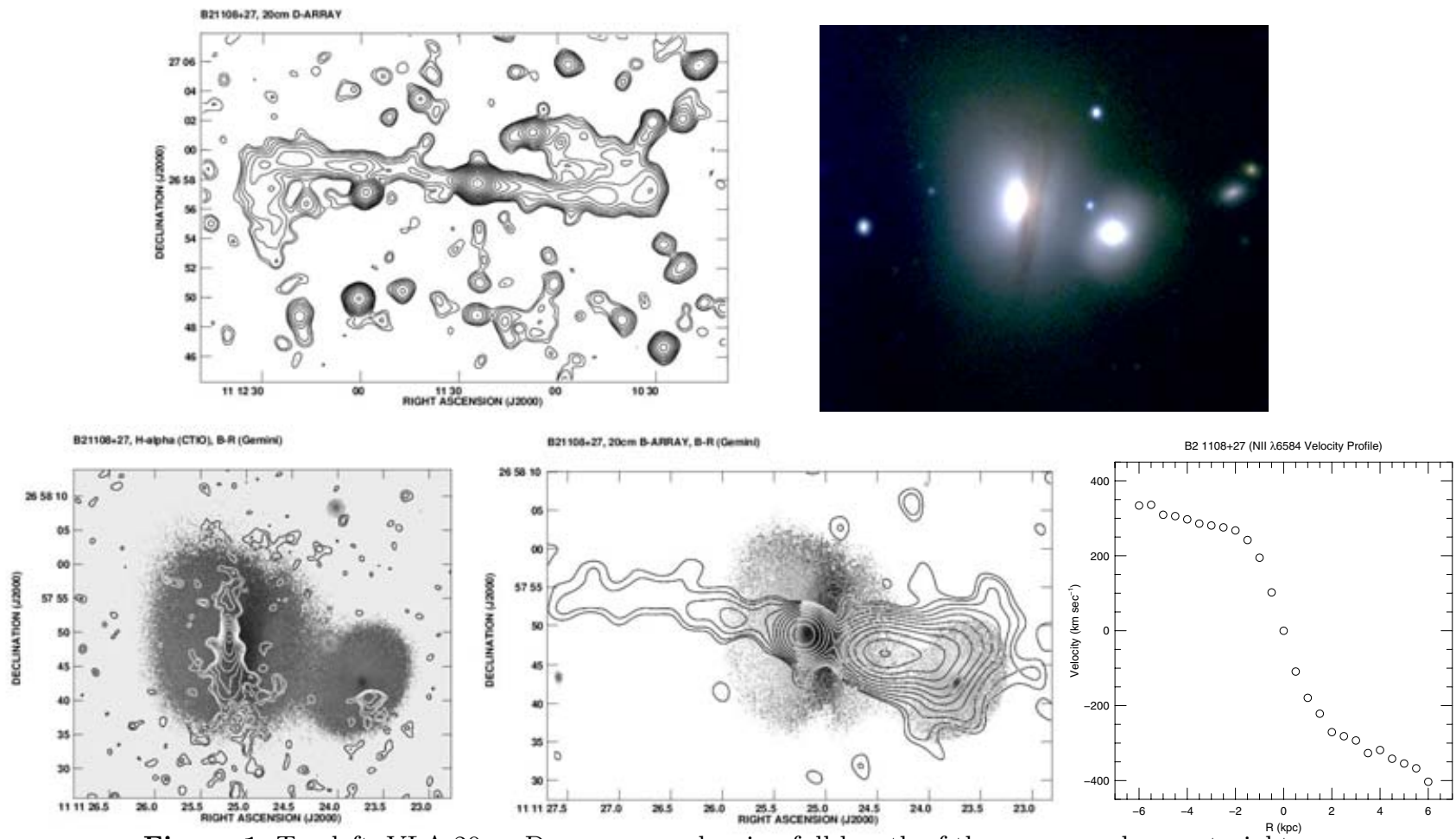

Figure 1. Top left: VLA $20 \mathrm{~cm}$ D-array map showing full-length of the source and very straight jets, Top right: BVR image from Gemini-South with the Acquistion camera and an image quality of 0.8 arcsec. Bottom left: $H \alpha+[N I I]$ narrow-band image from CTIO (countours) overlayed on B-R color map. Note the dust-lane parallel to the extended emission-line gas. Middle: Inner radio jets overlayed on B-R color map, Right: rotation profile measured from [NII] from GMOS-S longslit spectrum.
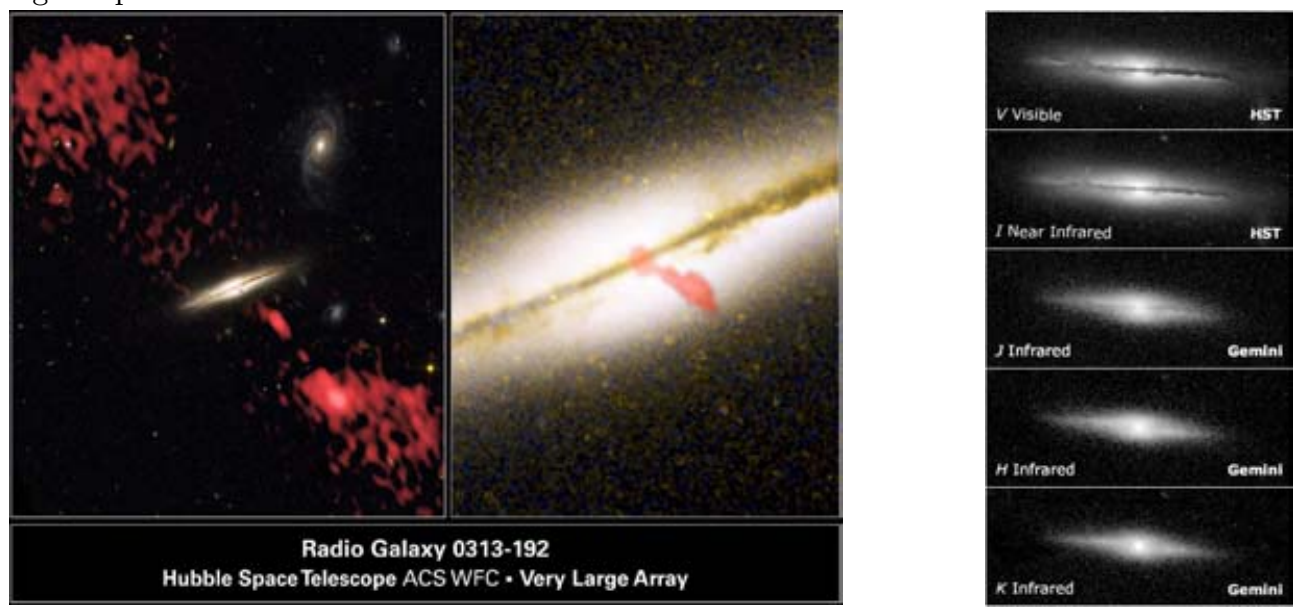

Figure 2. Left: HST/ACS + VLA overlay of 0313-192. Right: HST V,I-band and Gemini-S Flamingos-I, J,H,K images $(\mathrm{IQ}=0.39$ arcsec $)$

\section{References}

Keel, W. C., Ledlow, M. J., \& Owen, F. N. 2002, BAAS, 34, 1245

Ledlow, M. J., Owen, F. N., Yun, M. S., \& Hill, J. M. 2001, ApJ, 552, 120

Ledlow, M. J., Owen, F. N., \& Keel, W. C. 1998, ApJ, 495, 227 